\title{
Relação entre a Classificação Clínica de White e a Histopatologia das Placentas de Gestantes Diabéticas
}

\author{
The Relationship between White's Classification and the Histopathological \\ Changes in the Placentas of Diabetic Pregnant Women
}

\begin{abstract}
Iracema de Mattos Paranhos Calderon ${ }^{1}$, César Pereira Lima ${ }^{2,3}$, Marilza Vieira Cunha Rudge ${ }^{1}$
\end{abstract} Gilberto De Napoli ${ }^{2}$, Emílio Antonio Jeckel Neto ${ }^{4}$, Maria Delgi Ramos ${ }^{1}$

\section{RESUM0}

Objetivo: analisar a relação entre a classificação clínica de White e as alterações histopatológicas de placentas de gestantes diabéticas, comparando, de forma qualitativa, as alterações histopatológicas de placentas de gestantes não-diabéticas e diabéticas gestacionais (classes Ae $A / B$ ), clinicas de curta duração (classes $B$ e C) e clinicas com vasculopatia (classes $D$ a FRH), no termo e no pré-termo, e de acordo com a qualidade do controle glicêmico na gestação.

Pacientes e Métodos: foram colhidas amostras de placentas de todas as gestantes diabéticas, atendidas entre 1991 e 1996 na Maternidade do Hospital das Clinicas da Faculdade de Medicina de Botucatu, coradas pela técnica de hematoxilina-eosina e submetidas a exame histopatológico. A qualidade do controle glicêmico foi analisada pela média glicêmica da gestação e classificada em adequada e inadequada, com limite de $120 \mathrm{mg} / \mathrm{dl}$. A idade da gestação foi individualizada em termo e pré-termo.

Resultados: observou-se que 42 recém-nascidos (43,3\%) eram de termo e o restante, de prétermo (56,7\%). O indice de prematuridade foi maior nas diabéticas clinicas (classes B e C; $D$ a FRH). Algumas alterações histopatológicas só foram encontradas em placentas de gestantes diabéticas: degeneração cistóide, edema corial, edema da intima, dismaturidade, hiperplasia das células de Hofbauer, vilite, células fantasmas, dois vasos no cordão umbilical e endarterite. Conclusões: as alterações histopatológicas de placentas de gestantes com diabete gestacional (classes A e A/B), clínico de curta duração (classes B e C) e clinico com vasculopatia (classes $D$ a FRH) foram semelhantes às das não-diabéticas e, portanto, independeram da classificação clinica de White. As alterações histopatológicas de placentas de gestantes diabéticas não se relacionaram com a idade gestacional ao nascimento e com a qualidade do controle glicêmico materno. A comparação entre as alterações histopatológicas e a elevada proporção de recémnascidos pré-termo nas diabéticas clinicas, classes $D$ a FRH, sugerem amadurecimento placentário precoce nas diabéticas clínicas com vasculopatia.

PALAVRAS-CHAVE: Diabetes mellitus. Placenta. Prematuridade.

\section{Introdução}

Inúmeros estudos morfológicos placentá-

\footnotetext{
${ }^{1}$ Faculdade de Medicina de Botucatu - SP

${ }^{2}$ Fundação Faculdade Federal de Ciências Médicas de Porto Alegre - RS

${ }^{3}$ Faculdade de Medicina da PUC-RS / Porto Alegre - RS

${ }^{4}$ Instituto de Geriatria e Gerontologia da PUC-RS / Porto Alegre - RS

Correspondência:

Iracema de Mattos Paranhos Calderon

Rua Atílio Losi, 226 - Jardim Paraíso

18610-260 - Botucatu - SP
}

rios foram realizados na tentativa de explicar a fisiopatologia do comprometimento fetal no diabete. O problema é que as alterações encontradas não são características das placentas das diabéticas ${ }^{1-6}$. As alterações macroscópicas dependem da severidade do diabete materno, pois a análise da literatura ${ }^{7-9}$ mostra correlação entre a classificação de White ${ }^{10}$ e os critérios morfológicos da placenta ${ }^{11}$. Entretanto, são discordantes os resultados dos trabalhos correlacionando a gravidade do diabete materno, pela 
classificação de White $^{10}$, com a patologia placentária. Isso pode ser decorrente da heterogeneidade dos fatores maternos e fetais, que resultariam em alterações da estrutura ou função placentárias. Whitsett e Brownscheidle ${ }^{2}$ destacaram que lesões placentárias semelhantes podem ocorrer em gravidez normal, e sugeriram que as diferenças deveriam ser mais quantitativas do que qualitativas.

Nas gestantes diabéticas sem vasculopatia, as placentas são maiores, mais pesadas, com aumento do número de células, provavelmente devido ao aumento na velocidade de divisão celular, que ocorre antes da 36 a semana. Quando a angiopatia é grave, podem ser encontrados infartos a partir de 32-34 semanas $^{12-14}$.

No diabete existe imaturidade estrutural e funcional da placenta em relação à idade gestacional, que inclui tamanho aumentado do vilo terminal, aparência edematosa, diminuição da vascularização e da formação da membrana vásculo-sincicial, citotrofoblasto residual e aumento das células de Hofbauer ${ }^{15}$. O significado deste citotrofoblasto metabolicamente ativo no final da gestação é controverso: não está claro se representa retardo na maturação do órgão ou regressão a um estadio celular mais primitivo, induzido por alteração degenerativa do sinciciotrofoblasto.

al-Okail \& al-Attas ${ }^{16}$ não conseguiram correlacionar as alterações estruturais placentárias em pacientes com diabete de longa duração e gestacional mal controlado, comparadas às não diabéticas. Jones e Fox ${ }^{15,17}$ demonstraram, por estudos ultra-estruturais e histoquímicos, que as alterações morfológicas placentárias do diabete gestacional são semelhantes às descritas para as insulino-dependentes, porém em menor quantidade. Essas alterações, mais quantitativas do que qualitativas, entre as diabéticas gestacionais e clínicas, reforçam a hipótese de que a doença materna pode produzir uma gama de alterações no meio micrometabólico da placenta ${ }^{2}$.

O objetivo deste trabalho é analisar a influência da classificação clínica de White sobre as alterações histopatológicas de placentas de gestantes diabéticas, comparando, de forma qualitativa, as alterações histopatológicas de placentas de gestantes não-diabéticas e diabéticas gestacionais (classes A e A/B), clínicas de curta duração (classes $B$ e C) e clínicas com vasculopatia (classes D a FRH), no termo e no pré-termo, e de acordo com a qualidade do controle glicêmico na gestação.

\section{Pacientes e Métodos}

É um estudo baseado na análise histopatológica, prospectivo e cego, de placentas provenientes de gestantes normais e diabéticas, acompanhadas no Serviço de Diabete e Gravidez da Disciplina de Obstetrícia da Faculdade de Medicina de Botucatu - UNESP. A análise histopatológica foi realizada no Departamento de Patologia da Fundação Faculdade Federal de Ciências Médicas de Porto Alegre. Foram colhidas amostras de placentas de todas as gestantes diabéticas $(\mathrm{n}=91)$ atendidas no período de 1991 a 1996 e de seis gestantes não diabéticas.

Foram incluídas no estudo as placentas de pacientes com diagnóstico de diabete clínico, das classes B a FRH, e de diabete gestacional, das classes $\mathrm{A}$ e $\mathrm{A} / \mathrm{B}^{10}$. O grupo não-diabético foi composto por placentas de pacientes com rastreamento positivo para diabete sem confirmação diagnóstica (grupo IA) ${ }^{18}$.

As gestantes e suas placentas foram classificadas segundo White ${ }^{10}$ e agrupadas, de acordo com Gabbe ${ }^{19}$, em diabéticas gestacionais (classes A e A/B), diabéticas clínicas de curta duração (classes B e C) e diabéticas clínicas com vasculopatia (classes D a FRH).

A qualidade do controle glicêmico materno foi analisada pela média glicêmica da gestação (MG), calculada pelo somatório de todas as glicemias plasmáticas avaliadas nos perfis glicêmicos de 24 horas, dividido pelo total de dosagens realizadas. O controle glicêmico foi considerado adequado quando a $\mathrm{MG}$ foi igual ou inferior a $120 \mathrm{mg} / \mathrm{dl}$ e inadequado quando superior a este limite ${ }^{20}$. Após o parto, as placentas foram classificadas como sendo de gestação termo e pré-termo, com limite de idade gestacional, respectivamente, superior e inferior a 37 semanas completas.

Imediatamente após a dequitação, foi colhido um fragmento central do cotilédone, no local da inserção do cordão umbilical na placenta, desde a face materna até a face fetal, e um fragmento proximal do cordão umbilical. Ambos foram fixados em formol e confeccionadas as lâminas, coradas pela técnica de hematoxilina-eosina (HE).

Num estudo piloto, a análise de 20 placentas de gestantes diabéticas identificou 22 alterações histopatológicas, descritas a seguir:

Degeneração cistóide: presença de lacunas acelulares, contendo material fluido, proteináceo, encontradas no cório e, eventualmente, no vilo.

Edema corial: aumento de líquido ao nível 
corial.

Edema da intima: túnica íntima dos vasos espessada.

Hemorragia intersticial: extravasamento de sangue para o tecido extravascular.

Congestão: vasos repletos de sangue.

Infarto subcorial: hemorragia em área subcorial.

Edema vilositário: identificado pelo espaçamento das células, pelo acúmulo excessivo de líquido extravascular e extracelular distendendo o vilo, com distribuição irregular.

Fibrose do vilo ou intervilosa: caracterizada pelo aumento de colágeno no interior do vilo ou como organização de áreas de infarto.

Calcificação: achados corados pela $\mathrm{HE}$ em azul escuro, sem tecido placentário identificável em seu interior, comuns em áreas mortas ou zonas nas quais o metabolismo está diminuído. Ocorrem como manifestações do processo distrófico. É um tecido degenerado onde o cálcio se precipita.

Degeneração focal hialina: é identificada pela presença de massas homogêneas, amorfas e eosinofílicas, de uma substância fortemente refringente, tingivel com corantes ácidos. A patogenia não está totalmente clara. Discute-se a possibilidade de precipitação, por fibrinólise parcial ou imperfeita, ou absorção das proteínas do colágeno nos processos inflamatórios ou alterações circulatórias como conseqüência da acidose cística.

Dismaturidade: vilos com características de imaturidade entremeados com vilos maduros.

Hiperplasia das células de Hofbauer: aumento das células de Hofbauer ao nivel dos vilos.

Corioangiose: caracterizada por aumento do número dos vasos vilositários em extensão variável do disco placentário, ao nível dos cotilédones. Pode ser compensatória, decorrente de insuficiente aporte sanguineo.

Nós sinciciais: denominação para a especialização do trofoblasto que se caracteriza por aglomerado nuclear com projeções periféricas dos vilos. Fazem parte da estrutura da placenta e têm coloração escura. É índice de maturação placentar e tem diminuição das células do citotrofoblasto.

Vilite: presença de exsudato inflamatório comprometendo os vilos.

Amnionite focal: exsudato inflamatório ao nível do âmnio.

"Células fantasmas" ou restos celulares: células mortas, com desaparecimento do núcleo, restando apenas o contorno celular.
Dois vasos: ausência de uma artéria no cordão umbilical.

Endarterite: oclusão vascular, geralmente por proliferação endotelial ou conjuntiva, conseqüente a processos inflamatórios vasculares ou na vizinhança de vasos.

Membrana duplicada: espessamento de membrana basal pelo alargamento do espaço sub-celular.

Hemorragia de cordão: presença de hemácias na geléia de Wharton.

Tais alterações foram agrupadas pelo patologista, de acordo com a etiopatogenia, em:

lesões circulatórias: degeneração cistóide, edema da intima, hemorragia intersticial, congestão e infarto subcorial.

lesões degenerativas: edema vilositário, fibrose do vilo ou intervilosa, calcificação e degeneração focal hialina.

lesões proliferativas: dismaturidade, hiperplasia das células de Hofbauer, corioangiose e nós sinciciais.

lesões inflamatórias: vilite e amnionite focal.

outras lesões: incluindo "células fantasmas" ou restos celulares, dois vasos, endarterite, membrana duplicada e hemorragia de cordão (Figuras 1 a 5 ).

O exame histopatológico do material foi cego por parte do patologista, que não tinha conhecimento prévio da classe diabética e da $\mathrm{MG}$ da paciente.

$\mathrm{Na}$ análise individual das placentas, foram atribuídos escores para a presença (1) ou ausência (0) das alterações histopatológicas. O somatório destes escores, dentro das classes de gestantes diabéticas, relacionado à idade gestacional (termo e pré-termo) e à qualidade do controle glicêmico (adequado e inadequado), foi usado para calcular o escore total.

$O$ indice (\%) de alteração placentária (IAP) foi calculado pela seguinte fórmula: IAP (\%)=Escore total . 22 . n . 100; onde 22 é número de alterações histopatológicas observadas nas placentas de gestantes diabéticas (estudo piloto) e n é o número de placentas estudadas por grupo.

A ocorrência de alterações histopatológicas foi analisada pelo teste do $\chi^{2}$, empregando-se, quando necessário, o teste exato de Fisher. As médias de glicemia materna e peso do recémnascido foram comparadas pelo teste " $t$ " de Student. Adotou-se 5\% como nível de significância estatística $(p<0,05)$.

Este trabalho foi aprovado pelo Comitê de Ética em Pesquisa da Faculdade de Medicina de Botucatu. 

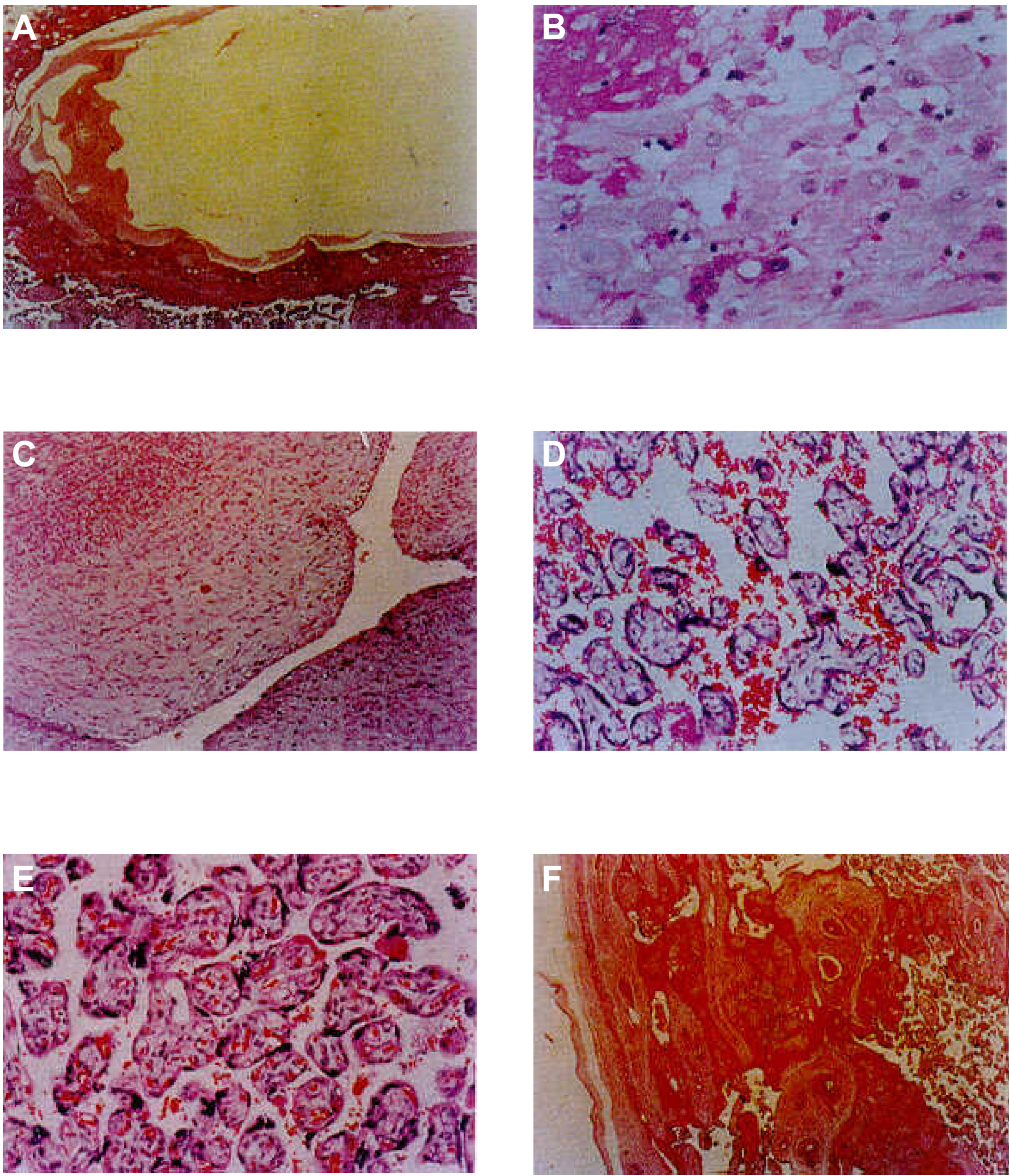

Figura 1 - Alteraç̃es circulatórias: A) degeneração cistóide: lacunas acelulares, com material fluido, proteináceo, encontradas no cório e, eventualmente, no vilo (HE lupa); B) edema corial: aumento de líquido ao nível corial (HE 40X); C) edema da íntima: túnica íntima dos vasos espessada (HE 10X); D) hemorragia intersticial: extravasamento de sangue para o tecido extra-vascular (HE 20X); E) congestão: vasos repletos de sangue (HE 20X); F) infarto sub-corial: hemorragia em área sub-corial (HE lupa). 

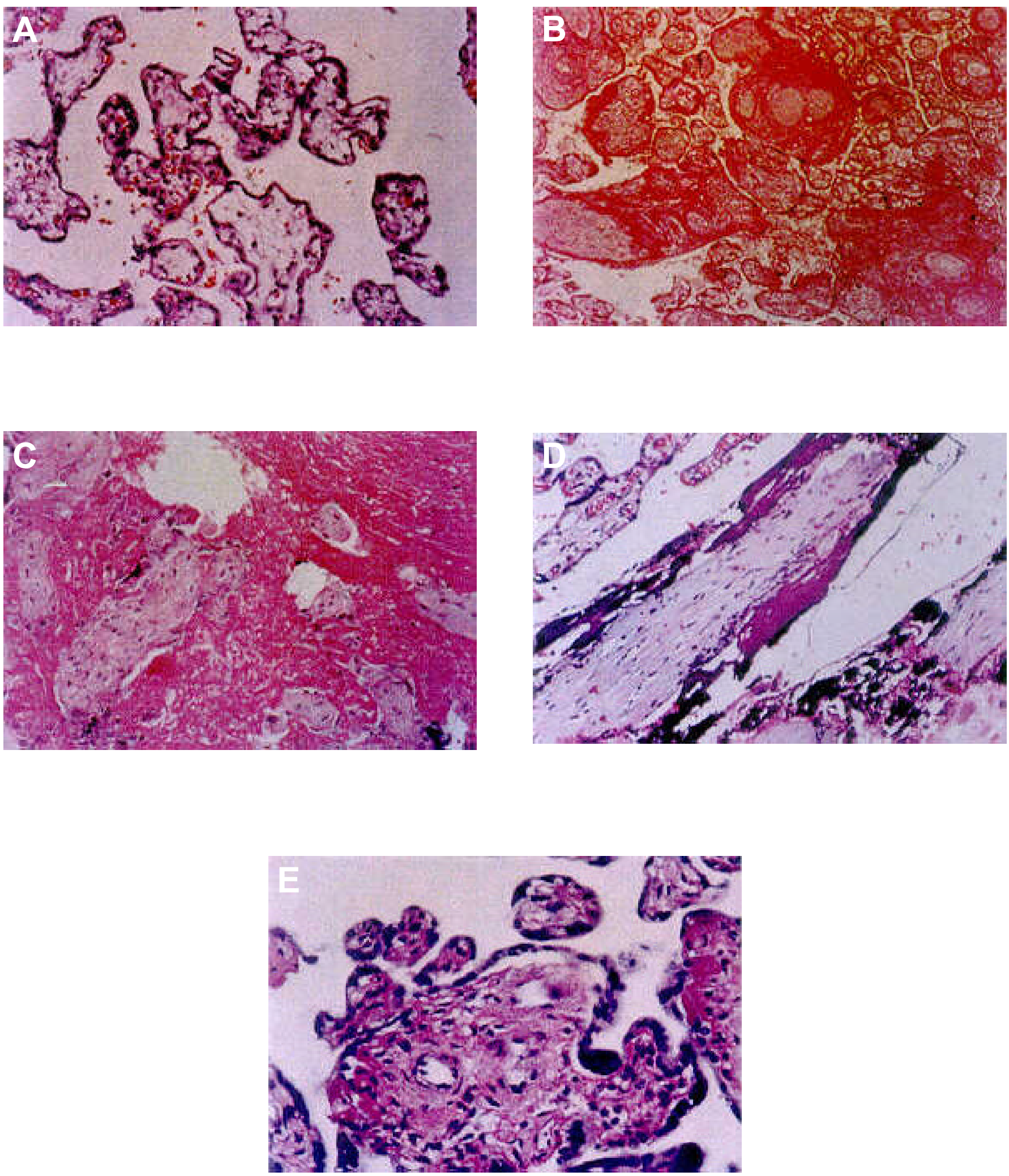

Figura 2-Alterações degenerativas: A) edema vilositário: identificado pelo espaçamento das células, pelo acúmulo excessivo de líquido extravascular e extracelular distendendo o vilo, com distribuição irregular (HE 20X); B e C) fibrose do vilo (HE 10X) ou intervilosa (HE 20X): caracterizada pelo aumento de colágeno no interior do vilo ou como organização de áreas de infarto; D) calcificação: achados corados pela HE em azul-escuro, sem tecido placentári identificável em seu interior, comuns em áreas mortas ou zonas nas quais o metabolismo está diminuído. Ocorrem como manifestações do processo distrófico. E um tecido degenerado onde o cálcio se precipita (HE 20X); E) degeneração focal hialina: é identificada pela presença de massas homogêneas, amorfas e eosinofilicas, de uma substância fortemente refringente, tingivel com corantes ácidos. A patogenia não está totalmente clara. Discute-se a possibilidade de precipitação, por fibrinólise parcial ou imperfeita, ou absorção das proteínas do colágeno nos processos inflamatórios ou alterações circulatórias como consequêencia da acidose cística (HE 40X). 

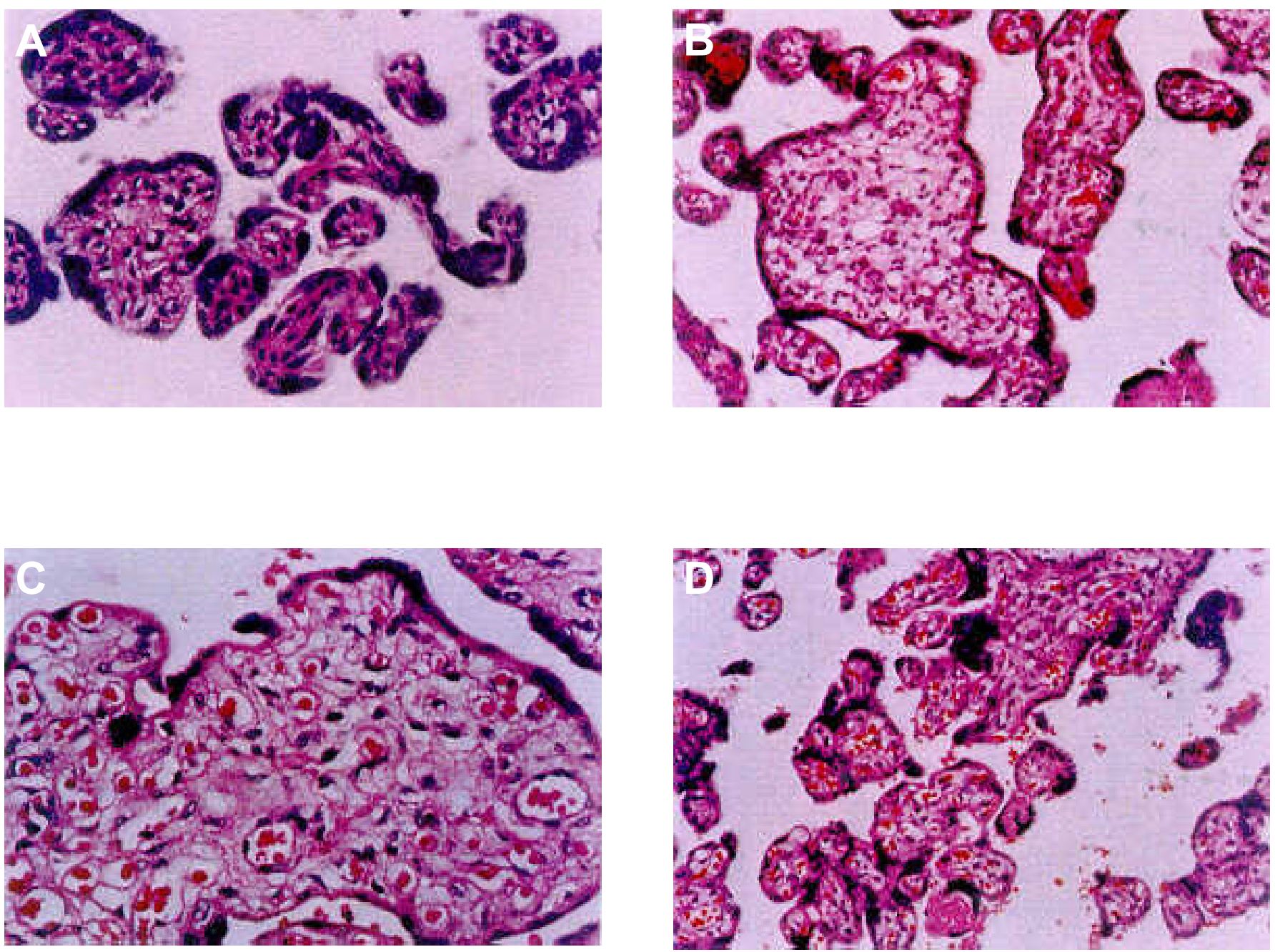

Figura 3 - Alterações proliferativas: A) dismaturidade: vilos com características de imaturidade entremeados com vilos maduros (HE 40X); B) hiperplasia das células de Hofbauer: aumento das células de Hofbauer ao nível dos vilos (HE 20X); C) corioangiose: caracterizada por aumento do número dos vasos vilositários em extensão variável do disco placentar, ao nivel dos cotilédones. Pode ser compensatória, decorrente de insuficiente aporte sanguíneo (HE 40X); D) nós sinciciais: denominação para a especialização do trofoblasto que se caracteriza por aglomerado nuclear com projeções periféricas dos vilos. Fazem parte da estrutura da placenta e têm coloração escura. É índice de maturação placentar e tem diminuição das células do citotrofoblasto (HE 20X).
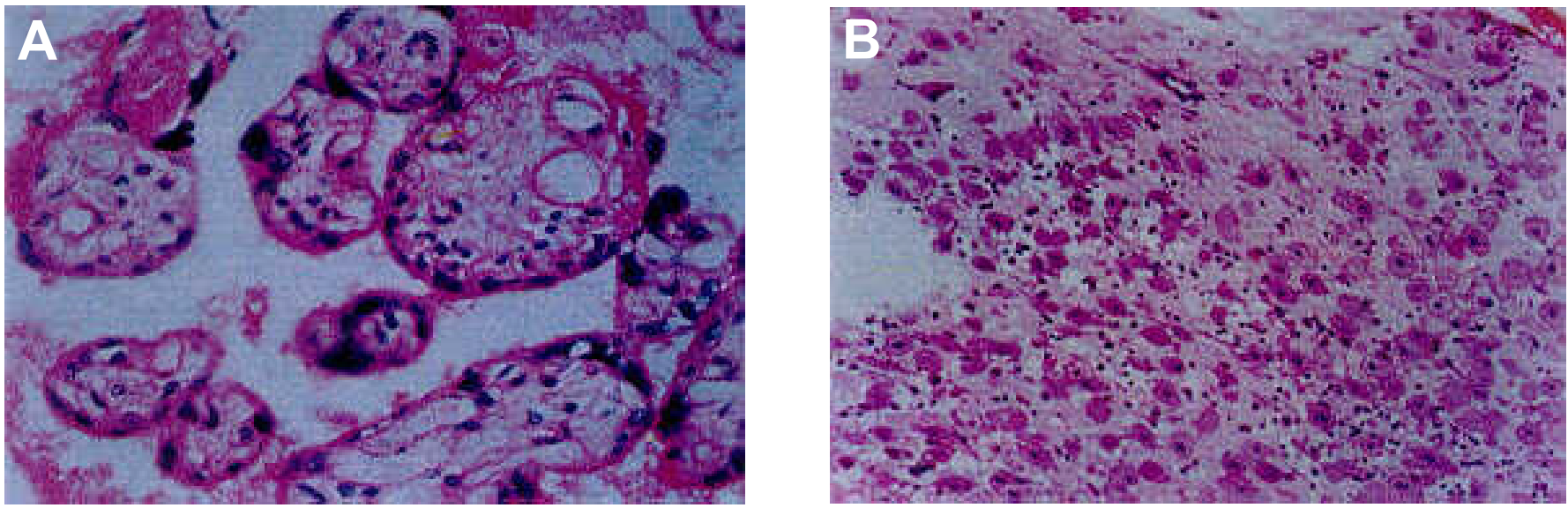

Figura 4 - Alterações inflamatórias: A) vilite: presença de exsudato inflamatório comprometendo os vilos (HE 40X); B) amnionite focal: exsudato inflamatório ao nivel do âmnio (HE 20X). 

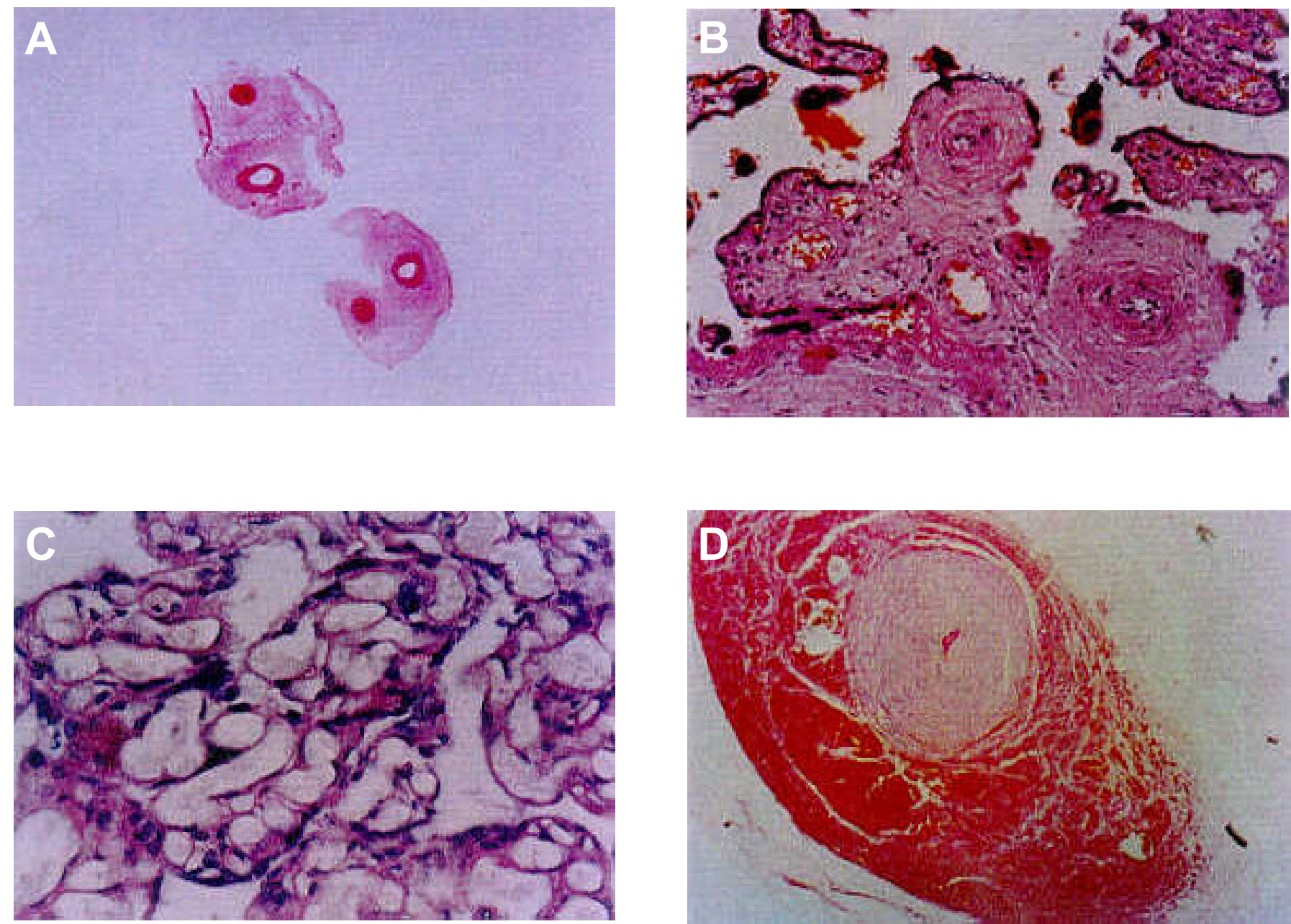

Figura 5-Outra alterações: A) dois vasos: ausência de uma artéria no cordão umbilical (HE lupa); B) endarterite: oclusão vascular, geralmente por proliferação endotelial ou conjuntiva, conseqüente a processos inflamatórios vasculares ou na vizinhança de vasos (HE 20X); C) membrana duplicada: espessamento de membrana basal pelo alargamento do espaço sub-celular (HE 40X); D) hemorragia de cordão: presença de hemácias na geléia de Wharton (HE lupa).

\section{Resultados}

Foram analisadas 97 gestações, classificadas de acordo com White ${ }^{10}$ e agrupadas segundo Gabbe ${ }^{19}$ : não-diabéticas ( $\mathrm{n}=6$ ), diabéticas gestacionais (classes A e A/B - $\mathrm{n}=36$ ), diabéticas clinicas de curta duração (classes B e C - $\mathrm{n}=$ 43) e diabéticas clínicas com vasculopatia (classes D a FRH - $\mathrm{n}=12$ ). A idade materna foi semelhante entre os grupos, variando entre 26 e 29 anos completos. As pacientes diabéticas gestacionais apresentaram MG superior às nãodiabéticas e inferior às das diabéticas clínicas. Entre as diabéticas clínicas, o tempo de evolução e a vasculopatia não interferiram nos valores da MG.

Os menores pesos de recém-nascidos foram observados nas classes D a FRH. Nas demais classes de gestantes diabéticas, os recémnascidos tiveram média de peso equivalente aos dos filhos de mães não-diabéticas. Na classifica- ção de recém-nascidos, relacionando peso e idade gestacional, não se observou diferença entre as proporções de adequados, pequenos e grandes, sendo a maioria deles de peso adequado para a idade gestacional (AIG). Observou-se que 42 recém-nascidos $(43,3 \%)$ eram de termo e o restante, de pré-termo $(56,7 \%)$. O índice de prematuridade foi maior nas diabéticas clínicas (classes B e C; D a FRH) (Tabela 1).

Algumas alterações histopatológicas só foram encontradas em placentas de gestantes diabéticas: degeneração cistóide, edema corial, edema da intima, dismaturidade, hiperplasia das células de Hofbauer, vilite, células fantasmas, dois vasos no cordão umbilical e endarterite (Tabela 2). Outras, puderam ser observadas tanto em placentas de gestantes diabéticas como nãodiabéticas, mas apenas algumas mostraram diferença significativa. Quando se considerou a classificação clínica de White $^{10}$ e o agrupamento de Gabbe $^{19}$, o infarto subcorial foi mais freqüente em placentas de gestantes normais, comparadas com 
placentas de diabéticas gestacionais (classes A e A/B). A fibrose intervilosa diferenciou as placentas de diabéticas gestacionais e clinicas de curta duração (classes A e A/B > B e C). A membrana duplicada foi mais observada em placentas de gestantes não-diabéticas quando comparadas com as diabéticas gestacionais (A e A/B) e clinicas de curta duração (B e C) (Tabela 2).

Tabela 1 - Características maternas e dos recém-nascidos (RN) e média glicêmica da gestação (MG) de não-diabéticas e diabéticas gestacionais (classes Ae A/B), clínicas de curta duração (classes Be C) e clínicas com vasculopatia (classes D a FRH).

\begin{tabular}{|c|c|c|c|c|}
\hline \multirow[b]{2}{*}{ Características } & \multirow[b]{2}{*}{ Não-diabéticas } & \multicolumn{3}{|c|}{ Diabéticas (classes) } \\
\hline & & $A$ e $A / B$ & $\mathrm{~B} \mathrm{e} C$ & D a FRH \\
\hline \multicolumn{5}{|l|}{ Maternas } \\
\hline \multicolumn{5}{|l|}{ Recém-nascidos } \\
\hline Peso médio $\left.(g)^{*}\right)$ & 3250 & 3417 & 3229 & 2458 \\
\hline Total de casos & 6 & 36 & 43 & 12 \\
\hline
\end{tabular}

Tabela 2 - Porcentagem (\%) de placentas com alterações histopatológicas nas gestantes não-diabéticas e diabéticas gestacionais (classes Ae A/B), clínicas de curta duração (classes Be C) e clínicas com vasculopatia (classes D a FRH).

\begin{tabular}{|c|c|c|c|c|}
\hline \multirow[b]{2}{*}{ Alterações Histopatológicas } & \multirow[b]{2}{*}{ Não-Diabéticas } & \multicolumn{3}{|c|}{ Classes de diabéticas } \\
\hline & & $A$ e $A / B$ & & D a FRH \\
\hline \multicolumn{5}{|l|}{ Circulatórias } \\
\hline Degeneração cistóide & & $2(5,6)$ & $1(2,3)$ & $1(8,3)$ \\
\hline Edema corial & & & $1 \quad(2,3)$ & $1(8,3)$ \\
\hline Edema da íntima & & $3(8,3)$ & $1 \quad(2,3)$ & \\
\hline Hemorragia intersticial & $1(16,7)$ & $1(2,8)$ & $2 \quad(4,7)$ & \\
\hline Congestão & $2(33,3)$ & $21(58,3)$ & $28(65,1)$ & $6(50,0)$ \\
\hline Infarto sub-corial $\left({ }^{*}\right)$ & $2(33,3)$ & $1(2,8)$ & $2 \quad(4,6)$ & \\
\hline \multicolumn{5}{|l|}{ Degenerativas } \\
\hline Edema vilositário & $1(16,7)$ & $8(22,2)$ & $8(18,6)$ & $5(41,7)$ \\
\hline Fibrose do vilo & $1(16,7)$ & $5(13,9)$ & $5(11,7)$ & $1(8,3)$ \\
\hline Fibrose intervilosa $\left(^{*}\right)$ & $3(50,0)$ & $32(88,9)$ & $30(69,8)$ & $9(75,0)$ \\
\hline Calcificação & $4(66,7)$ & $17(47,2)$ & $22(51,7)$ & $4(33,3)$ \\
\hline Degeneração focal hialina & $4(66,7)$ & $33(91,7)$ & $34(79,1)$ & $10(83,3)$ \\
\hline \multicolumn{5}{|l|}{ Proliferativas } \\
\hline Dismaturidade & & $6(16,7)$ & $2(4,6)$ & $3(25,0)$ \\
\hline Hiperplasia Hofbauer & & & $1(2,3)$ & \\
\hline Corioangiose & $1(16,7)$ & $2(5,6)$ & $5(1,7)$ & \\
\hline Nós sinciciais & $1(16,7)$ & $3(8,3)$ & $4(9,3)$ & $1(8,3)$ \\
\hline \multicolumn{5}{|l|}{ Inflamatórias } \\
\hline Vilite & & & $1(2,3)$ & $1(8,3)$ \\
\hline Amnionite focal & $1(16,7)$ & $5(13,9)$ & $3(7,0)$ & \\
\hline \multicolumn{5}{|l|}{ Outras } \\
\hline Células fantasmas & & & $1(2,3)$ & \\
\hline Dois vasos & & & $2(4,6)$ & $1(8,3)$ \\
\hline Endarterite & & $5(13,9)$ & $2(4,6)$ & $1(8,3)$ \\
\hline Membrana duplicada $\left(^{*}\right)$ & $2(33,3)$ & & & $1(8,3)$ \\
\hline Hemorragia do cordão & $3(50,0)$ & $7(19,4)$ & $5(11,7)$ & $2(16,7)$ \\
\hline Total de casos & 6 & 36 & 43 & 12 \\
\hline
\end{tabular}

(*) Significância estatística $(\mathrm{p}<0,05)$ :

Infarto subcorial: Não-diabéticas > A e A/B; Fibrose intervilosa: $A$ e A/B > B e C; Membrana duplicada: Não-diabéticas > A e A/B $=B$ e C 
Nas classes de gestantes avaliadas, o IAP foi semelhante em todas as placentas e não diferenciou as de termo e pré-termo. O mesmo se observou em relação à qualidade do controle glicêmico (MG adequada e inadequada). O limite de $\mathrm{MG}$ de $120 \mathrm{mg} / \mathrm{dl}$ não diferenciou as alterações histopatológicas placentárias nas classes de gestantes diabéticas (Tabela 3).

Tabela 3 - Freqüência (\%), escore total e índice de alterações placentárias (IAP), agrupadas conforme a etiopatogenia, em gestantes não-diabéticas e diabéticas, relacionadas à idade gestacional eà qualidade do controle glicêmico: adequado e inadequado.

\begin{tabular}{|c|c|c|c|c|c|}
\hline \multirow[b]{2}{*}{ Alterações Histopatológicas } & \multirow{2}{*}{$\begin{array}{c}\text { Não-diabéticas } \\
\text { Termo } n=6\end{array}$} & \multicolumn{2}{|c|}{ Diabéticas } & \multicolumn{2}{|c|}{ Diabéticas } \\
\hline & & Termo $\mathrm{n}=36$ & Pré-Termo $n=55$ & MG adeq $n=68$ & MG inadeq $n=23$ \\
\hline Circulatórias & $5(19,3)$ & $29(18,7)$ & $42(20,7)$ & $55(19,8)$ & $16(19,8)$ \\
\hline Proliferativas & $2(7,7)$ & $14(9,0)$ & $13(6,4)$ & $22(8,0)$ & $5 \quad(6,2)$ \\
\hline Inflamatórias & $1(3,8)$ & $4(2,6)$ & $6(3,0)$ & $7 \quad(2,5)$ & $3(3,6)$ \\
\hline Escore total & 26 & 155 & 203 & 277 & 81 \\
\hline $\operatorname{IAP}(\%)^{(*)}$ & 19,7 & 19,6 & 16,8 & 18,5 & 16,0 \\
\hline
\end{tabular}

$\left(^{*}\right) \mathrm{ns}(\mathrm{p}>0,05)$

\section{Discussão}

Vários estudos demonstraram que as placentas de diabéticas não têm achados histopatológicos patognomônicos ${ }^{3-6,21}$. Outros trabalhos relataram elevada incidência de anomalias nas placentas, porém sem definir achado característico $^{22}$. Vogel $^{23}$ descreveu a placopatia diabética, caracterizada por vilo embriônico persistente, dismaturidade, desordens de ramificação do vilo e corioangiose. Fox $^{22}$ encontrou anomalias na maturação do vilo, endarterite obliterativa intravilosa, espessamento da característica membrana basal do trofoblasto e necrose fibrinóide do vilo corial.

Das 22 alterações histopatológicas avaliadas neste trabalho, nove foram encontradas apenas nas placentas de gestantes diabéticas. A dismaturidade esteve presente em 11 de 91 $(12,1 \%)$ e a endarterite, em cerca de $9 \%$ das placentas de gestantes diabéticas. Essas alterações foram consideradas, por outros autores, como lesões freqüentes em placentas de gestantes diabéticas $^{20}$.

As placentas das gestantes diabéticas mostraram características histopatológicas semelhantes às das não-diabéticas, sendo que algumas características que apresentaram diferença estatística não foram suficientes para separar as placentas das gestantes não-diabéticas das diabéticas gestacionais ou clínicas, dificuldade salientada, também, por Haust ${ }^{24}$.

Chama a atenção que, a despeito das semelhanças entre a incidência de lesões histopa- tológicas em placentas de gestantes não-diabéticas e diabéticas, a fibrose intervilosa foi mais freqüente em diabéticas gestacionais do que em diabéticas clínicas de curta evolução. Isto pode estar relacionado ao diagnóstico, que é mais tardio no diabete gestacional e, em conseqüência, o início do tratamento é retardado. Este aspecto clínico torna-se importante, pois a fibrose leva à hipoxia fetal, complicação associada a índices baixos de Apgar e aumento da morbimortalidade neonatal ${ }^{25}$, evidenciando a importância do diagnóstico e tratamento precoces do diabete gestacional.

As lesões degenerativas e circulatórias foram as mais comuns, tanto nas placentas das diabéticas como das não-diabéticas. Os estudos pioneiros sobre placentas de gestantes diabéticas já caracterizavam a pletora, que se relaciona tanto à congestão e hemorragias, como à hipóxia tecidual, levando à corioangiose ${ }^{25}$. As alterações degenerativas dos vilos - edema, fibrose, calcificação e degeneração hialina - foram interpretadas por esses autores como imaturidade do órgão. Entretanto, estudos posteriores admitem a dismaturidade da placenta no diabete, associada à corioangiose, alterações vilositárias e aumento dos nós sinciciais ${ }^{6,23,24}$, características observadas, também, em placentas de gestantes não-diabéticas.

As lesões proliferativas também não foram exclusivas do diabete materno. A corioangiose e o aumento dos nós sinciciais, conseqüentes à hipoxia tecidual, podem estar presentes na hipertensão, na doença hemolitica perinatal e em tabagistas crônicas, o que explicaria o encontro 
dessas alterações histopatológicas nas placentas de gestantes não-diabéticas.

O IAP traduziu o percentual das características histopatológicas nas placentas de gestantes não-diabéticas e diabéticas, no termo e pré-termo e no controle glicêmico materno adequado e inadequado. Apesar do cuidado na diferenciação destas variáveis confundidoras, o IAP não diferenciou as placentas resultantes de diferentes condições clínicas, idade gestacional ou qualidade de tratamento.

al-Okail \& al-Attas ${ }^{16}$ encontraram alterações mais acentuadas nas placentas das diabéticas gestacionais, comparadas às das clinicas de longa evolução. Entretanto, não conseguiram correlacionar as alterações estruturais placentárias provenientes de pacientes com diabete de longa duração com as de diabete gestacional mal controlado. Teasdale ${ }^{26}$ mostrou que diferentes alterações estruturais placentárias provêm de pacientes com a mesma gravidade clínica da doença.

Fox $^{22}$ e Haust ${ }^{24}$ já chamavam atenção para o cuidado que deve ser tomado no exame das placentas, ou seja, comparar placentas de mesma idade gestacional. Whitsett e Brownscheidle ${ }^{2}$ aventaram a hipótese de que a dismaturidade representaria retardo na maturação ou regressão a um estadio celular mais primitivo. Mesmo aceitando que a imaturidade estrutural e funcional da placenta é alteração que pode ser observada em gestações complicadas pelo diabete em idades gestacionais mais avançadas, não foi possivel diferenciar as alterações histopatológicas, no termo e pré-termo, entre diabéticas e nãodiabéticas. É interessante a concordância dos achados histopatológicos do grupo controle e do grupo com diabete clínico com vasculopatia, uma vez que as placentas do grupo controle são todas de termo, ao contrário daquelas de diabéticas clínicas com vasculopatia, nas quais $91,7 \%$ são pré-termo. Pode-se inferir que as placentas resultantes de gestações das classes D a FRH no pré-termo são semelhantes às das gestantes normais no termo. Este achado sugere senescência precoce das placentas no diabete materno com vasculopatia.

É necessário que se analise com precaução os trabalhos que mostram resultados histopatológicos diferentes nas placentas de gestantes diabéticas. Nos últimos tempos, houve mudança na abordagem do diabete materno e, conseqüentemente, nos resultados perinatais. $\mathrm{O}$ rígido controle da glicemia materna, principio básico do tratamento atual no diabete, previne as complicações decorrentes da hiperglicemia nos meios materno, fetal, neonatal e placentário ${ }^{20}$.
Isto também aconteceu neste trabalho, em que a faixa média de glicemia materna não foi muito diferente entre os grupos e não atingiu $120 \mathrm{mg} /$ dl. Na opinião de Benirschke e Kaufmann ${ }^{27}$, nenhuma das alterações é específica para o diabete materno, refletindo apenas a concentração de glicose disponivel e os ajustes fetais para a oferta intermitente e excessiva deste metabólito. Os resultados aqui encontrados reforçam tal opinião - a histopatologia placentária não tem características específicas do diabete e não se relaciona com a gravidade do quadro clínico materno. Concluímos que as alterações histopatológicas de placentas de gestantes com diabete gestacional (classes A e A/B), clínico de curta duração (classes $\mathrm{B}$ e C) e clínico com vasculopatia (classes $\mathrm{E}$ a FRH) foram semelhantes às das não-diabéticas e, portanto, independeram da classificação clínica de White. As alterações histopatológicas de placentas de gestantes diabéticas não se relacionaram com a idade gestacional e com a qualidade do controle glicêmico materno.

A comparação entre as alterações histopatológicas e a elevada proporção de recém-nascidos pré-termo nas diabéticas clínicas, classes $\mathrm{D}$ a FRH, sugerem amadurecimento placentário precoce nas diabéticas clínicas com vasculopatia.

\section{SUMMARY}

Purpose: to analyze the relationship between White's classification and the histopathological, changes occurring in the placentas of diabetic pregnant women, performing a qualitative comparison of histopathological changes in the placentas of nondiabetic pregnant women with those in diabetic ones (classes $A$ and $A / B$ ), clinical, short duration (classes $B$ and $C$ ), and clinical with vasculopathy (classes D to FRH), studying the influence of the quality of glycemic control and of gestational age on placental changes in the three groups of diabetic pregnant women.

Patients and methods: specimens of placentas were collected from all diabetic pregnant women seen between 1991 and 1996 in the Maternity Section of the Hospital das Clínicas, Faculdade de Medicina de Botucatu, stained using the hematoxylin-eosin technique, and submitted to a histopathological examination. The quality of glycemic control was analyzed by the glycemia average of gestation and classified as adequate or inadequate, with a limit of $120 \mathrm{mg} / \mathrm{dl}$. Gestational age was individualized as term and preterm.

Results: forty-two newborns (43.3\%) were born at term and the remaining were preterm (56.7\%). The prematurity rate was higher for women with clinical diabetes (classes $B$ and $C ; D$ to FRH). Some histopathological alterations were observed only in placentas from diabetic pregnant 
women: cystoid degeneration, chorial edema, intima edema, dysmaturity, Hofbauer cell hyperplasia, villitis, ghost cells, two vessels in the umbilical cord, and endarteritis.

Conclusions: histopathological changes in the placentas of pregnant women with gestational diabetes (classes $A$ and $A / B)$, clinical, short duration (classes $B$ and $C$ ), and clinical with vasculopathy (classes $D$ to FRH) were similar to those in the nondiabetic ones, and, therefore, were independent of White's clinical classification. The histopathological changes in the placentas of pregnant women with gestational diabetes (classes $A$ and $A$ and $B)$, clinical, short duration (classes $B$ and $C$ ), and clinical with vasculopathy (classes $D$ to $F R H$ ) were not related to gestational age at birth and to the quality of glycemic control of the mother. The comparison between histopathological changes and the increased number of preterm newborns in clinical diabetes, class D to FRH, suggest early placental ageing in clinical diabetes patients.

KEY WORDS: Diabetes. Placenta. Prematurity.

\section{Referências}

1. Oh W. Neonatal care and long-term outcome in infants of diabetic mothers. In: Merkatz IR, Adam PAJ. The Diabetic Pregnancy: a perinatal perspective. $1^{\text {st }}$ ed. New York: Grune $\&$ Stratton; 1979. p.195-205.

2. Whitsett JA, Brownscheidle CM. Aspects of placental structure and function in maternal diabetes. In: Merkatz IR, Adam AJ. The Diabetic Pregnancy: a perinatal perspective. New York: Grune \& Stratton; 1979. p.123-43.

3. Driscoll SG. The pathology of pregnancy complicated by diabetes mellitus. Med Clin North Am 1965;49:1053-67.

4. Benirschke K, Driscoll SG. The pathology of the human placenta. $1^{\text {st }}$ ed. Berlin: Springer-Verlag; 1967. p.512.

5. Jácomo KH, Benedetti WL, Sala MA, Alvarez H. Pathology of the trophoblast and fetal vessels of the placenta in maternal diabetes mellitus. Acta Diabetol Lat 1976; 13:216-35.

6. Singer DB. The placenta in pregnancies complicated by diabetes mellitus. Perspect Pediatr Pathol 1984; 8:199-212.

7. Emmrich P, Gödel E. Morphologie der Plazenta bei mütterlichem Diabetes mellitus. Ergebnisse morphologischer Untersuchungen. Zentralbl Allg Pathol 1972;116:56-63.

8. Emmrich P. Morphologie der Plazenta bei mütterlichem Diabetes mellitus. I. Vorschlag einer einheitlichen morphologischen Plazentadiagnostik unter Berücksichtigung internistischer, geburtshilflicher und pädiatrischer Gesichtspunkte sowie Literaturübersicht. Zentralbl Gynäkol 1972; 94:881-7.
9. Vogel M, Kloss K. Diabetes in der Schwangerschaft: neue morphologische Befunde Plazenta und Fetus in perinataler Medizin bd. 6.7. Dtsch. Kongress für Perinatale Medizin; Berlin 1974.

10. White P. Classification of obstetric diabetes. Am J Obstet Gynecol 1978; 130:228-30.

11.Semmler K, Emmrich P. Morphologie der Plazenta in Relation zur Glykämielage in der Schwangerschaft beim Diabetes mellitus. Z Geburtshilfe Perinatol 1989; 193:124-8.

12.Benirschke K, Brown WH. Vascular anomaly of umbilical cord: Absence of one umbilical artery in umbilical cords of normal and abnormal fetuses. Obstet Gynecol 1955; 6:399-404.

13.Benirschke K. A review of the pathologic anatomy of the human placenta. Am J Obstet Gynecol 1962; 84:1595-622.

14.Driscoll SG. Placental manifestations of malformation and infection. In: Gruenwald P, editor. The Placenta and its Maternal Supply Line: effects of insufficiency on the fetus. $1^{\text {st }}$ ed. Baltimore: University Park; 1975. p.248.

15.Jones CJ, Fox H. An ultrastructural and ultrahistochemical study of the placenta of the diabetic woman. J Pathol 1976; 119:91-9.

16.al-Okail MS, al-Attas OS. Histological changes in placental syncytiotrophoblasts of poorly controlled gestational diabetic patients. Endocr J 1994; 41:355-60.

17.Jones CJ, Fox H. Placental changes in gestational diabetes. An ultrastructural study. Obstet Gynecol 1976; 48:274-80.

18.Rudge, MVC. Diabetes e gestação. Femina 1988; 16:125-40.

19.Gabbe SG. Management of diabetes mellitus in pregnancy. Am J Obstet Gynecol 1985; 153:824-8.

20.Rudge MVC, Calderon IMP, Ramos MD, Abbade JF, Rugolo LMSS. Perinatal outcome of pregnancies complicated by diabetes and by maternal daily hyperglycemia not related to diabetes - A retrospective 10 years analysis. Gynecol Obstet Invest - in press.

21.Zacks SI, Blazar AS. Chorionic villi in normal pregnancy, pre-eclamptic toxemia, erythroblastosis, and diabetes mellitus: a light and electron microcoscope study. Obstet Gynecol 1963; 22:149-53.

22.Fox H. Pathology of the placenta in maternal diabetes mellitus. Obstet Gynecol 1969; 34:792-8.

23.Vogel M. Plakopathia diabetica. Entwicklungsstörungen der Plazenta bei Diabetes mellitus der Mutter. Virchows Arch Pathol Anat Physiol Klin Med 1967; 343:51-63.

24. Haust D. Maternal diabetes mellitus effects on the fetus and placenta. In: Naeye RL, Kissane JM, Kaufman N, editors. Perinatal Diseases. $1^{\text {st }}$ ed. Baltimore: Williams \& Wilkins; 1981. p.201-77.

25. Naeye RL. Disorders of the placenta, fetus and neonate: diagnosis and clinical significance. $1^{\text {st }}$ ed. St. Louis: Mosby Year Book; 1992. p.375.

26. Teasdale F. Histomorphometry of the human placenta in class B diabetes mellitus. Placenta 1983; 4:1-12.

27.Benirschke K, Kaufmann P. Pathology of the human placenta. $3^{\text {rd }}$ ed. New York: Springer- Verlag; 1995. p.871. 